\title{
Pioneering gene therapy on brink of approval
}

After two and a half years and three prior negative opinions, the Committee for Medicinal Products for Human Use (CHMP) of the European Medicines Agency (EMA) has now backed the approval of alipogene tiparvovec (Glybera, UniQure), an adeno-associated virus $(A A V)$ vector encoding lipoprotein lipase ( $L P L)$, for the treatment of LPL deficiency in patients with severe or multiple pancreatitis attacks. Approval by the European Commission, which is anticipated within the next 3 months, would make Glybera the first gene therapy to be approved outside China.

LPL deficiency is a rare autosomal recessive single gene deficiency, affecting about 1-2 people per million. It causes the build-up of lipoproteins in the gut wall following a meal and is associated with a number of complications, such as pancreatitis, which can be lifethreatening. Currently, there are no drugs to treat this condition.

"LPL is a great model for gene therapy since it is a recessive disease in which easily measured biomarkers can be used to assess the efficiency and stability of transgene expression," explains James Wilson, Professor of Pathology at the University of Pennsylvania, USA, who developed the AAV1 vector used in Glybera.

However, the rarity of the disease made clinical trials for this therapy especially challenging. Safety is also a key concern for gene therapy approaches in general - particularly with regards to potential immune reactions to the vector and insertional mutagenesis.

"Over 10 years, Glybera was tested in two observational studies and three interventional clinical studies, first in the Netherlands and then in Canada," says Daniel Gaudet, Professor at the University of Montreal, Canada, who led the clinical trials. "It was well tolerated by the majority of the 27 patients in the interventional studies, and local adverse reactions were found to be due to local LPL activity rather than anti-AAV immune responses," he adds. Barrie Carter, Past-President of the American Society for Gene and Cell Therapy, points out that AAV vectors, overall, have an impressive safety record. "The most notable feature about these vectors is

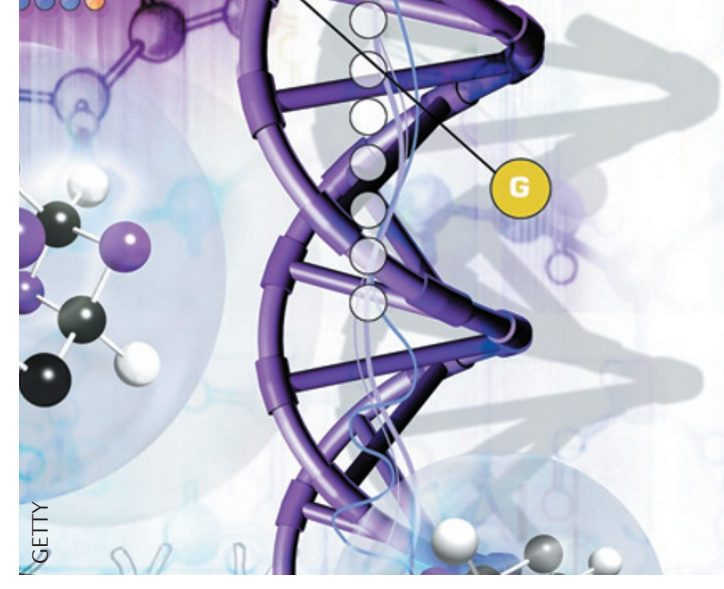

the fact that their genomes persist in the nucleus as episomes, leading to very long-term expression of the transgene, with extremely low integration rates into the nuclear DNA," he says.

A substantial number of other AAV-based products are in early clinical development, targeting other tissues or organs such as retina, liver and brain. UniQure is currently running five other gene therapy programmes, the most advanced of which involves an $\mathrm{AAV}$ vector encoding factor IX that has completed a Phase I/II trial for the treatment of haemophilia B.

Wilson thinks that the approval of Glybera will be huge in terms of encouraging further investment in gene therapy products. Gaudet adds that although the approval of Glybera will have limited impact clinically, it will have a substantial impact from a regulatory perspective: "It demonstrates that it is possible for a gene-based medication to be approved despite all the barriers, which include the complexity of the treatment, as well as many technical, clinical, social and ethical questions."

Alexandra Flemming 